\title{
A Comprehensive Study on Reactive and Proactive Routing Protocols under different performance Metric
}

\author{
Sadaf Wali ${ }^{1}$, Syed Irfan Ullah ${ }^{1}$, A.Wajid Ullah Khan ${ }^{1}$, Abdus Salam ${ }^{1}$
}

\begin{abstract}
Mobile Ad-hoc Network (MANET) has recently gained enormous studies attention because of their large potential of reliability and scalability in various fields. Besides, this Ad-hoc network is very flexible in nature which makes it more valuable for the researcher in the research area. Ad-hoc network provides better mobility due to which it uses dynamic topology. The routing protocols possess a very important role in any MANET. The routing protocols are divided into three big categories reactive protocols (on demand), proactive protocols (table-driven) and hybrid. The different routing protocols were created, which perform differently under different scenarios. This survey paper investigates the reactive and proactive routing protocol performance under different scenarios. The routing protocols like AODV, DSR, DSDV, AODV Modified (R-AODV) and TORA are compared under different parameters. The NS2 simulation tool is used for the simulation purpose. This paper shows the previous work done by the researchers in the field of reactive and proactive routing protocol based on its comparative analysis. Our results, contrarily to previously reported studies conducted on the same routing protocols, show the superiority of reactive over proactive protocols in routing such traffic under different network scenarios and parameters.
\end{abstract}

Keywords: $A O D V, D S R, D S D V$, TORA, R-AODV

\section{Introduction}

A Lot of work has been done in the area of routing protocols in recent time. Many routing protocols have been proposed for Ad-hoc community with attention on optimizing distinct aspects of the network routing. The Wireless Ad-hoc network is the collection of a group of mobile computers. The nodes help in packet forwarding and allow communication beyond its transmission range. A wireless Ad-Hoc network community is a decentralized form of wireless network which does no longer rely on a preexisting infrastructure. As wireless Ad-hoc network does not require a central device or control management. Therefore, it is easy to establish when needed. The routers in the network are free movers in any direction. Therefore, the topology is dynamic in nature. Every node in the wireless ad-hoc network may act both as an end consumer node or a router and could, therefore, trade its Link to different nodes often. It also enables other nodes to determine a path to another cell node and perform packet delivery that might not in the direct wireless communication. Ad-hoc provides flexible and big advantages in different environment such as military, commercial and technology. The interest of the researchers is increased for the Ad-hoc networks for a distributed set of applications. Applications such as gaming, military applications, commercial application and

${ }^{1}$ Department of computing and technology, Abasyn University, Peshawar, Pakistan. Corresponding Email:diyaaakhan098@gmail.com 
Sadaf Wali (et-al) A Comprehensive Study on Reactive and Proactive Routing Protocols under different performance

crises management services applications [1, 2].

As there is no specific device in this environment, therefore, the security challenges are the great concern for the researchers to work on it. Many demanding situations that still need to be solved in such regions as addressing, routing, place control, configuration management, interoperability, security, and excessive capacity technologies $[3,4]$. The routing is the procedure which is used to choose a path in a network. The main function of routing protocol is the selection of routes for multiple source destinations and its accurate delivery to the destination. In Ad-hoc networking the mobile nodes are used for searching the route or paths for the connection and sharing the data. The performance of any routing protocol is the end to end delay, throughput, PDF, NRL and Route Acquisition Time etc.

\section{Routing Protocols}

The Ad-hoc routing protocols are classified into three big classes

\subsection{Table-driven (proactive Algorithms)}

This Algorithm simply uses the tables. The data are stored and updated in these tables.

Examples are (DSDV), (WRP) wireless routing protocol (GSR) global state routing [4, 5].

\subsection{On Demand (reactive Algorithms)}

This Algorithm creates routes on demand bases by flooding the network with Route Request packets. The two key procedures of source initiated on demand-driven routing protocols are the route discovery process and route maintenance process. ABR, AODV, and $\mathrm{DSR}$ are reactive routing protocols.

\subsection{Hybrid protocols}

The composite of table driven and ondemand routing protocols which possess the attributes of both routing protocols together.
ZRP, CEDAR, and LAR routing protocols belongs to hybrid protocols [6].

In section 3 the protocols that are used in Ad-hoc are discussed. Section 4 describes comparison evaluation of routing protocols. Section 5 describes the simulation result of protocols. The last section presents the result and conclusion.

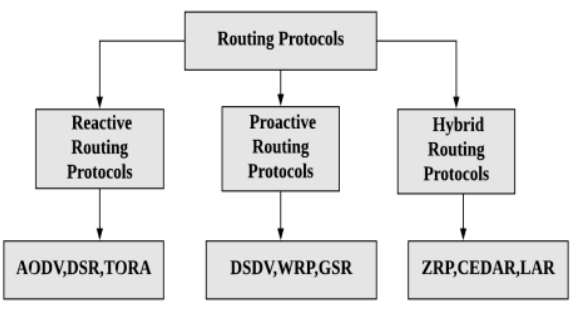

Fig 1. Different classes of routing protocols

\section{An Overview Of Protocols}

\subsection{Ad-hoc on-demand vector protocol (AODV)}

AODV is on demand protocol which creates the route on demand. The nodes move here and there very quickly which results in breaking links. The message mechanisms are having RREQs, RREPs, and RERRs. Route Maintenance and route discovery are used by AODV Whenever a sender wants to create a route its route discovery process can be done through flooding route request message. All the neighbors include in this process as they all get the route request messages. After reaching the destination route reply packet sends back on the same path used by RREQ packet. Each node maintains a sequence number and a routing table. The loop-free operation takes place in AODV which helps in repairing of breakage links. Whenever a link breaks, the affected nodes are informed about the infected or lost link [7, 8, 9, and 10]. 
Sadaf Wali (et-al) A Comprehensive Study on Reactive and Proactive Routing Protocols under different performance Metric (pp. 39-51)

3.2. Dynamic Source Routing Protocol (DSR)

This protocol is designed for the multi-hop networks of moving nodes. This is an efficient routing protocol which is based on demand routing protocol which has a self-organizing network. Working of this protocol can be illustrated in two steps Route identification and route Management. DSR guarantee loopfree routing. The source node will first check the route of the node in a cache to which it wants to send a packet. If it tries to find the route, it sends the packet. Otherwise it uses route discovery. After the destination received the packet, it stores the information contained by RREQs and sends a reply back to a source node. The route mechanism is responsible for checking whether the topology changes or not. In case of breakage of a link, that route is removed from cache route and informs all nodes by sending RERRs [12, 13, and 14].

\subsection{Destination- sequenced distance vector Protocol (DSDV)}

DSDV is a table-driven based on Bellmanford scheme. C. Perkins and P.Bhagwat developed DSDV in 1994. This algorithm helps in removing the routing loop problem. The records are recorded in a table. Every node entered in a table contains a sequence number. When the connection exists the series number is even else an odd number is used. The routing records sent between nodes with smaller updates usually.

Every node advertises routing information using broadcasting a routing table packet updated in case of network topology changes. Loop-Free and count to infinity problem is much reduced in DSDV [12, 13, and 14].

\subsection{Modified Ad-hoc on-demand vector protocol (R-AODV)}

It is the new version of AODV routing protocol with some modification in AODV. The R-AODV tries to manufacture a more dependable way between the source and destination keeping route stability under consideration. The change has been done in Hello and RREQ messages. In R-AODV new field is embedded by Hello message to record the sending time. At whatever point the (i+1)th node gets HELLO message sent from the ith node, it should record the HELLO messages arrival postpone time d_time. In route discovery stage the $(i+1)$ th node gathers the d_time values of hello message reached within the predefined period and makes count to these. At last, the $(i+1)$ th node appends them to the RREQ packet and surges/floods the bundles to the neighbors. At the point when the RREQ packet achieves the goal, the goal can choose a steady and solid route to the source as indicated by these measurements $[17,18]$.

\subsection{Temporally Ordered Routing Algorithm (TORA)}

It is an on-demand routing protocol which works on the concept of path reversal of the directed A-cyclic Graph (DAG). TORA searches multiple routes from sender to destination. TORA is a loop-free and bandwidth efficient protocol that's why TORA has the property to repair the route quickly during link breakage and provides multiple paths for the desired destination. It works well in networks where traffic increases gradually. TORA operate in the dynamic network. It uses Parameter "Height" to greatly emphasize the direction of a path between the nodes. TORA supports multicasting. TORA can be used in conjunction with Lightweight Adaptive Multicast Algorithm (LAM) to provide multicasting. The disadvantage of TORA is that the algorithm may also produce temporary invalid routes as in LMR.

Information in a network is managed in three different ways.

i. Route Erasure

ii. Route Discovery

iii. Route Maintenance

The protocol is able to detect the partition and erase all invalid routes [19, 20]. 
Sadaf Wali (et-al) A Comprehensive Study on Reactive and Proactive Routing Protocols under different performance

\section{Survey Of Routing Protocols} Performance Evaluation

The following performance metrics are used for performance investigation of protocols.

\subsection{Throughput}

To check the total rate of transmitted packets in a unit time in the network. The nodes received by the receiver per unit time.

$$
\text { Throughput }=\frac{(p r \times p s i z e)}{1000} \text { [3] }
$$

$p \boldsymbol{r}$ is used for the packet received and psize is the size of packets in bits.

\subsection{Average End -to- End delay}

The average time used by the sender to send the packets to the receiver. All the possible delays are included in it.

$$
\text { Average Delay }=\frac{\text { Total delay }}{\text { Count }}[3]
$$

$\mathrm{i}=$ packet sequence number

count $=$ Total packet count

delay[i] = receiving_time $[\mathrm{i}]-$

sending_time[i]

Total_Delay $=$ Total Delay + delay $[i]$

\subsection{NRL}

The total number of packets transmitted per data packet delivered at the destination.

$$
N R L=\frac{p c}{p d}
$$

Where $p c$ is the total control packets and the $p d$ is the total number of packets sent.

\subsection{PDR}

PDR is the ratio of a successful amount of data delivered to the destination sends by the source. PDR is used to measure the efficiency of protocols

$$
P D R=\frac{p r}{p s} \times 100[3]
$$

$p r$ is used for a packet received and $p s$ is used for a packet sent.

\section{Literature Review On Protocols}

\subsection{Throughput}

In the case of low mobility the throughput of AODV is higher than comparatively to DSR and DSDV. AODV gives better throughput than R-AODV. The DSDV performance is not very strong in a high mobility within the constant value 10 to 30 percent. The performance of TORA is better at high mobility but it has a lower throughput in other cases $[1,2,3,4,5]$.

\subsection{Packet Delivery Ratio (PDR)}

The packet delivery for DSR is the best among the protocols. The DSR is best in terms of Average PDR. AODV is having low PDR than DSR because it drops a higher number of packets. Packet loss of AODV is less than DSDV. DSDV performs worst for packet delivery. The packet loss issue is very sensitive in TORA compared to other routing protocols. TORA packet delivery is less than AODV. The PDR for Modified-AODV is the same as AODV but changes in velocity decrease rapidly in case of AODV. [1, 2, 3, 4, $5,19,20]$.

\subsection{End-to-End delay}

The Delay of AODV and DSR is more than DSDV. The routes are not shortest due to which AODV and DSR delay is poor. The TORA protocol is also having worst delay characteristics because of the loss of distance information with progress. The average delay of R-AODV is less than AODV 
Sadaf Wali (et-al) A Comprehensive Study on Reactive and Proactive Routing Protocols under different performance Metric

\subsection{Normalized Routing Load (NRL)}

The NRL for AODV is highest among the other routing protocols. DSDV has the least NRL which shows that DSDV is the best routing protocol for the optimal shortest path.

\section{Simulation Results And Analysis}

This section explains the simulation analysis carried out for the routing protocols. According to the different author's papers, the simulation results for the routing protocols are discussed below.

\subsection{PDR (Packet delivery ratio)}

A Wajid Khan [3] the result of PDR analysis for routing protocol describes that DSR works better under small network. DSR is greater than AODV. In the medium and larger network size, AODV works efficiently. Its PDR is greater. The reason behind it is that as the route discovery process is very quick, it allows the AODV to adapt to the route changes quickly.

Ajay Prakash [2] results show that while increasing pause time the PDF of AODV and DSDV is continuously decreasing for 10 numbers of a node. The results also describe that PDF of AODV is larger than DSDV.

Sapna S [5] according to their simulation result as the load will be less for less number of nodes DSR performs well. DSR performance slightly decreases as the number of nodes increased. DSDV performs better than the other two protocols with nodes increased. AODV also performs better but as nodes increase its performance slow down.

Anuj k [19] simulation result shows TORA is quite sensitive to packet loss than other protocols. The PDF of AODV is slightly less than DSR.

Humaira Nishat [18] papers compared the AODV and R-AODV. The result shows that the PDR for both the protocols is the same but with the increase of velocity, AODV ratio decreases. Whereas R-AODV remains the same.

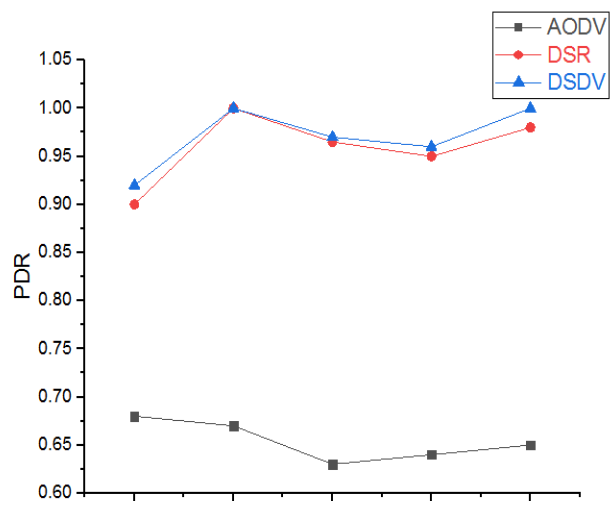

Fig 2: Packet delivery Ratio of AODV, DSDV, DSR

This graph shows the comparison of AODV, DSR, and DSDV under the PDR parameter. It shows that AODV performance is better as the packet delivery ratio is less than the other two protocols. The other two protocols packet delivery ratio is high.

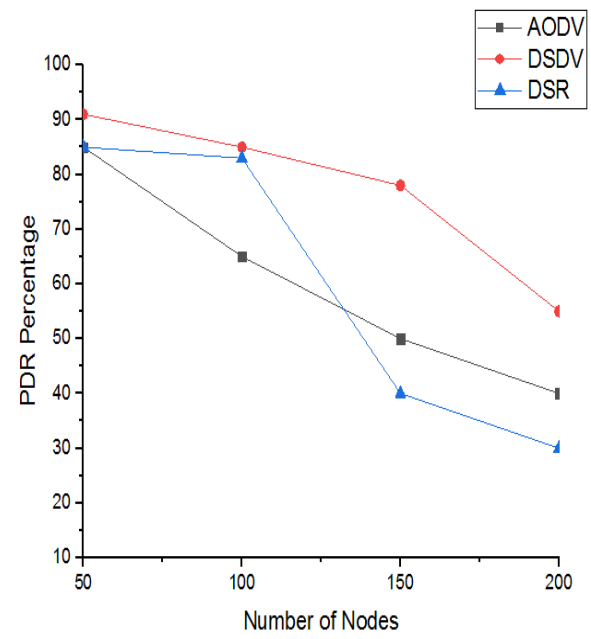

Fig 3: PDR of AODV, DSR, and DSDV 
In this graph 200 numbers of nodes are used for the comparison of AODV, DSR, and DSDV. The protocols were compared under different network sizes such as small, medium and large. DSR works better under small network size while for the large and medium size network AOD performs better.

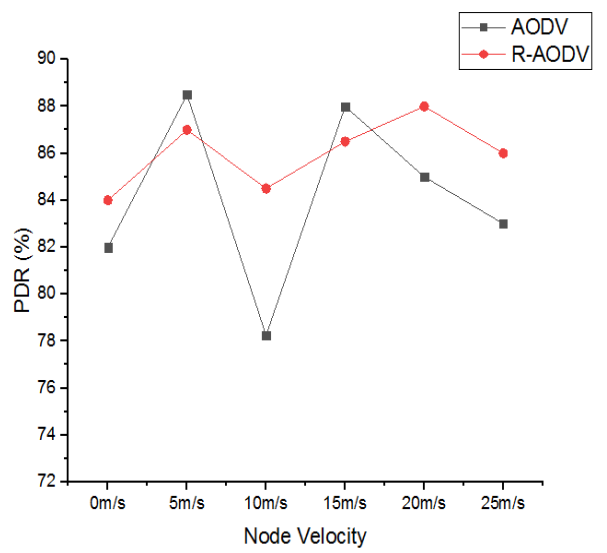

Fig 4: PDR of AODV and R-AODV

The graph shows that with the increase in node velocity AODV ratio decreases. The overall performance of both the protocols was the same.

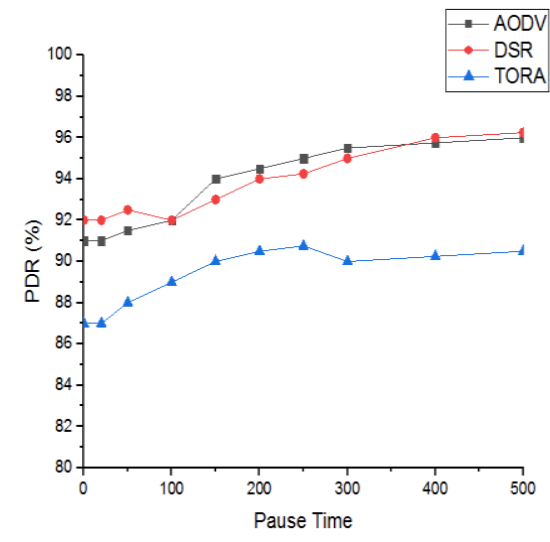

Fig 5: PDR of AODV, DSR, and TORA

The graph shows that AODV has lower PDR than DSR because of a high rate of packet dropping

\subsection{Throughput}

According to the paper of A Wajid khan [3], the throughput parameter is checked for AODV, DSR and DSDV routing protocols under different network scenarios like small, medium and large. The results show that the AODV protocol received more packets than the other two protocols. DSR throughput is not good in all networks. The reason behind the low performance is linked breakage.

Akshai Aggarwal [4] paper result shows that AODV performance is better under a large number of nodes, while DSR performance is better for a small number of nodes.

V. Rajeshkumar [9] this paper focuses on the comparison of three routing protocols such as AODV, DSR, and DSDV. The no of nodes was $(30,40$, and 50$)$. The overall performance of AODV was higher than the other two protocols. As AODV is loop-free therefore the routing packets for it is much more. DSR performs slightly low than AODV. Anuj k [19] paper describes that the overall performance of AODV is better. TORA performs well for high mobility rate while for low mobility its performance was low.

Humaira Nishat [18] the performance of both protocols was the same for the throughput but as the speed increases behind $18 \mathrm{~m} / \mathrm{s}$ R-AODV performance becomes low. 


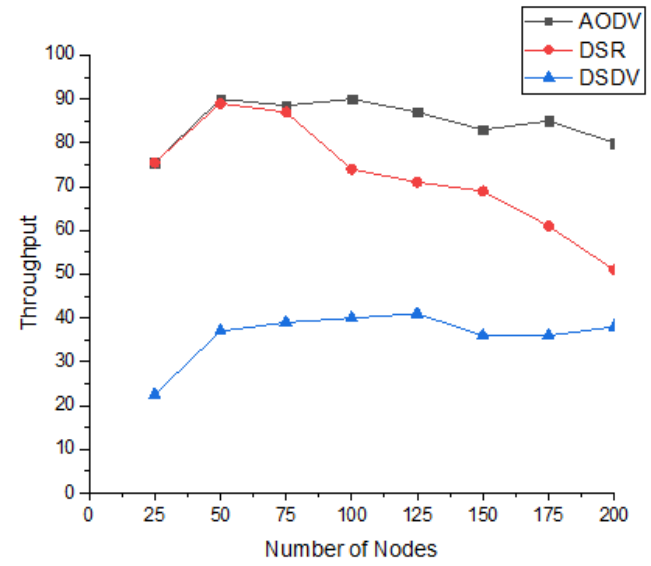

Fig 6:-Throughput for AODV, DSR, and DSDV

This graph represents the performance of protocols for the throughput parameter. According to this graph result, the throughput of AODV is high than other protocols.

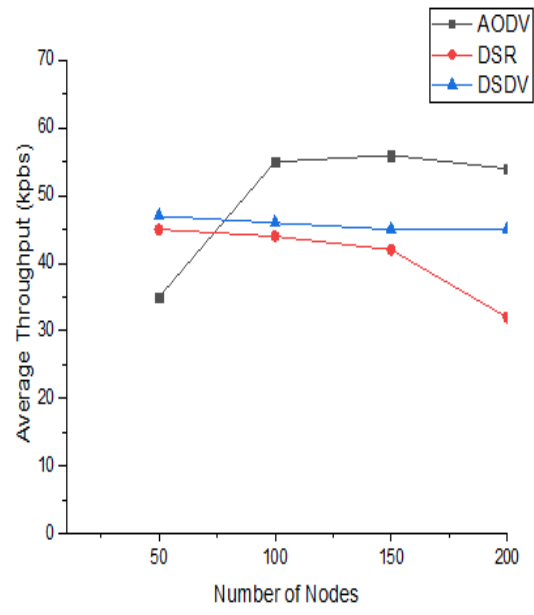

Fig 7: Average Throughput of AODV, DSR, and DSDV

The graph shows that AODV throughput is higher than DSR and DSDV

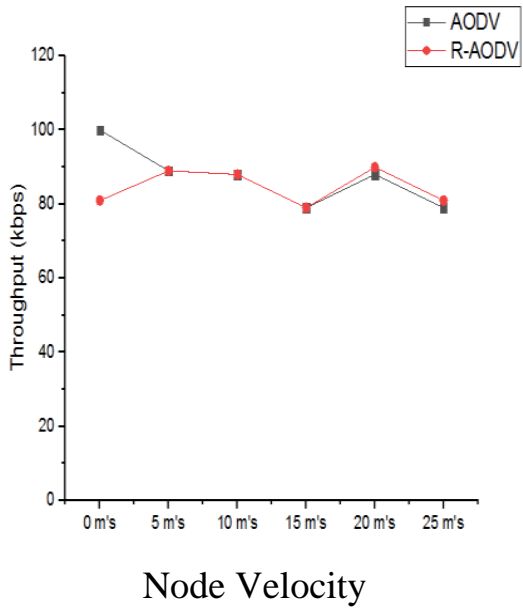

Fig 8: A throughput of AODV and R-AODV

The result of the graph shows that as the speed increases R-AODV performance becomes low. Both protocols result is almost the same.

\subsection{End-To-End Delay}

A Wajid khan [3] the average end to end delay of DSR is better than AODV. The AODV has very high delay under all network conditions. Ajay Prakash [2] the graphs show that the delay of AODV is much higher than DSDV. The reason behind the end to end delay is as AODV contains the address of next hop. During link breakage, the route discovery process Re-initiate which is waste of time.

Anuj k [19] the author compared AODV, DSR and TORA protocols. According to his simulation, the end to end delay was very poor for both AODV and DSR. DSR delay was slightly higher than AODV. The end to end delay decreases as the pause time increases. Similarly, TORA performance was also not very good. The reconstruction of a route was also not very quick which results in lengthy delays.

Humaira Nishat [18] the average delay of R-AODV is less than AODV. AODV gives more delay than R-AODV. 


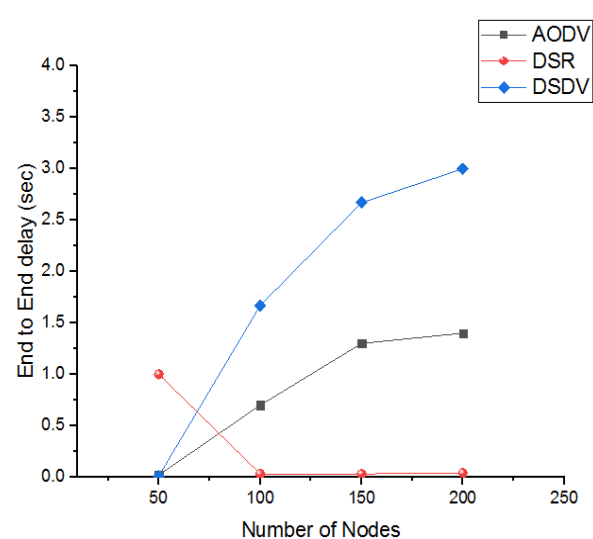

Fig 9: End to End Delay of AODV, DSR, and DSDV (with 10 connections)

The graph indicates the comparison of three protocols namely AODV, DSR, and DSDV. According to the result of the graph, the result of DSR is better than the other protocol.

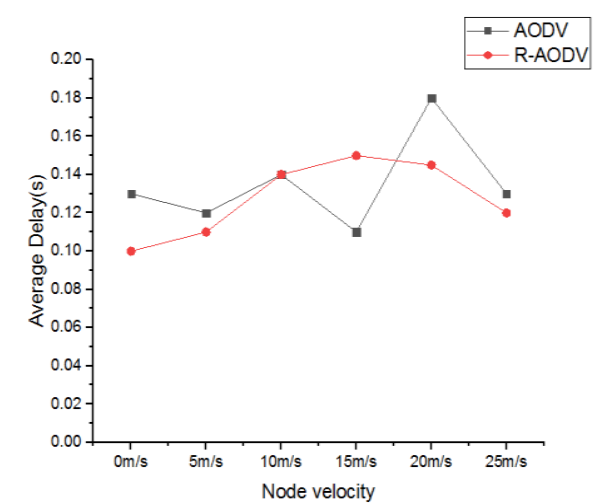

Fig 10: End to End delay of AODV and R-AODV

This graph compares the result for the AODV and R-AODV. The result of both the protocol shows that R-AODV performs better for the end to end delay

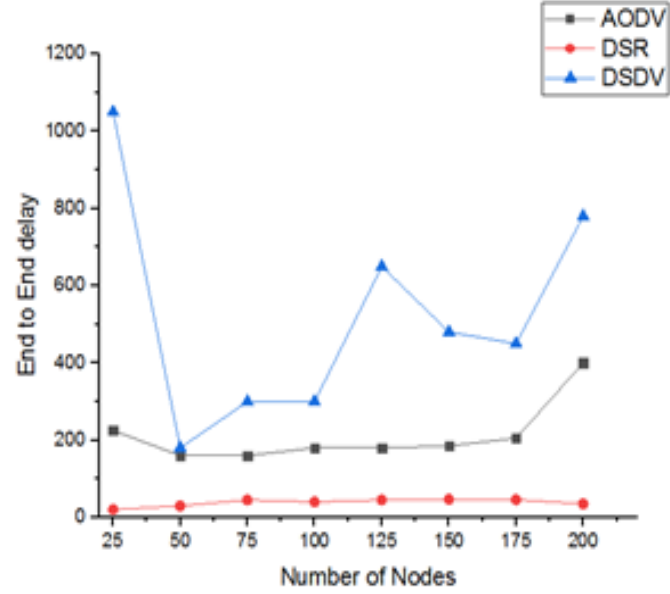

Fig 11: End to End delay of AODV, DSR and DSDV

The result of the graph shows that the average end to end delay of DSR is better than AODV and DSDV

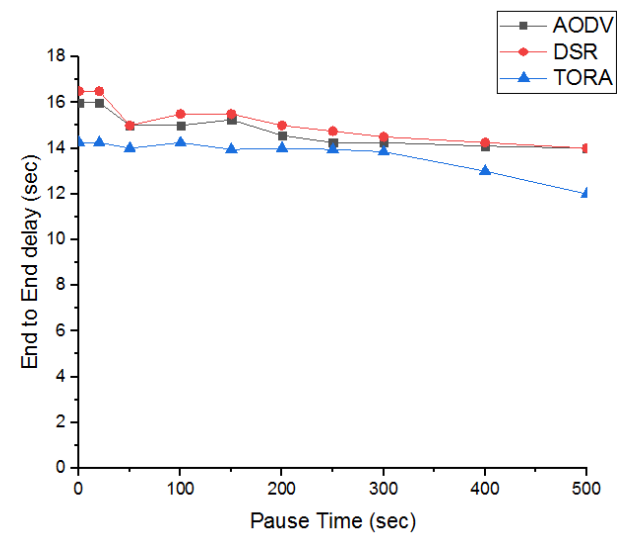

Fig 12: End to End Delay AODV, DSR and TORA

This comparative analysis is based on the protocols such as AODV, DSR, and TORA for the parameters end to end delay. According to the result, TORA performs better than AODV and DSR. 
Sadaf Wali (et-al) A Comprehensive Study on Reactive and Proactive Routing Protocols under different performance Metric

\subsection{NRL (Normalized routing load)}

A Wajid khan [3] the authors analyzed the performance of routing protocols for NRL. The result shows that AODV has low NRL in small and medium networks than DSR in a large network. Amirhossein Moravejosharieh[21] According to the author the DSR protocol has the best NRL than other protocols. In the value of density, DSDV has highest NRL while in speed density M-AODV has highest

NRL.

Guntupalli Lakshmikanth [22] according to their simulation result the NRL is 15 times increased in AODV and DSDV as the number of nodes increased from 40 to 100 . The DSR increased $25 \%$.

Geetha Jayakumar [23] According to the simulation result it shows that with the different variation of pause time DSR performs better than AODV. The NRL was low of DSR than AODV. AODV route discovery process takes more time than DSR. Therefore DSR has low NRL than AODV.

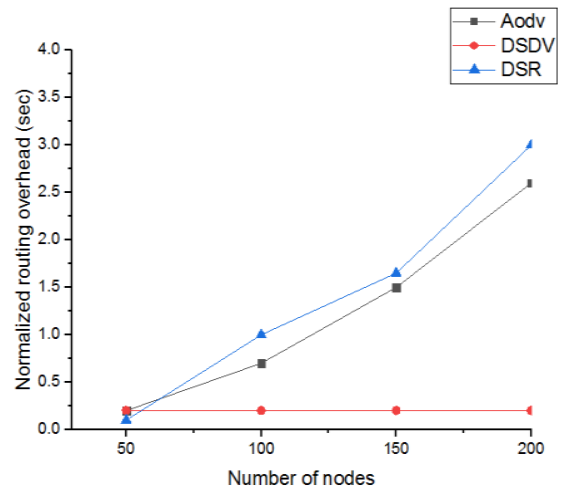

Fig 13: NRL of AODV, DSR, DSDV

This graph represents the performance of protocols for NRL which show that for both small and medium AODV has low NRL, while DSR performs better for large size.

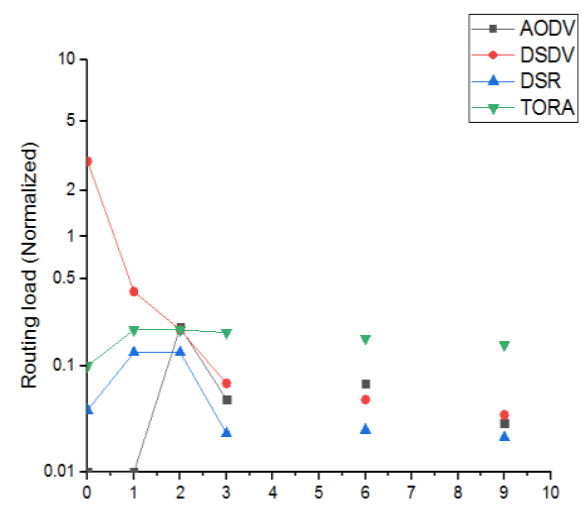

Fig 14:-NRL of AODV, DSDV, DSR, and TORA under low mobility case

This graph is based on the performance analysis of AODV, DSR, DSDV, and TORA which indicates that the DSR performs better than the other protocols. 
Sadaf Wali (et-al) A Comprehensive Study on Reactive and Proactive Routing Protocols under different performance

TABLE I. Comparative Analysis of Routing Protocols

\begin{tabular}{|c|c|c|c|c|}
\hline $\begin{array}{c}\text { PROTOCOL } \\
\text { PROPERTY }\end{array}$ & Avg AODV & Avg DSR & Avg DSDV & Avg TORA \\
\hline $\begin{array}{c}\text { Paper/Author } \\
\text { Name }\end{array}$ & Anuj k [19] & Anuj k[19] & Anuj k[19] & Anuj k[19] \\
\hline Throughput & N/A & N/A & N/A & N/A \\
\hline PDR & $95 \%$ & $95.5 \%$ & N/A & $90 \%$ \\
\hline $\begin{array}{c}\text { End to End } \\
\text { Delay }\end{array}$ & $16 \mathrm{sec}$ & $17 \mathrm{sec}$ & N/A & $14.25 \mathrm{sec}$ \\
\hline $\begin{array}{c}\text { Paper/Author } \\
\text { Name }\end{array}$ & $\begin{array}{c}\text { Samir } \\
\text { Robert[24] }\end{array}$ & $\begin{array}{c}\text { Samir Robert } \\
{[24]}\end{array}$ & $\begin{array}{c}\text { Samir Robert } \\
{[24]}\end{array}$ & $\begin{array}{c}\text { Samir Robert } \\
{[24]}\end{array}$ \\
\hline NRL & $0.3 \mathrm{sec}$ & $0.1 \mathrm{sec}$ & $3 \mathrm{sec}$ & $0.3 \mathrm{sec}$ \\
\hline
\end{tabular}

The table indicates the average result for the following routing protocols under different parameters. The results are based upon the paper of A Wajid khan [3]. The total number of nodes used in the simulation process is $(50,100,150,200)$.
The simulation time is $100 \mathrm{sec}$. packet size is 512 and interval time is 0.1 . The overall performance of AODV is better for all Parameters

TABLE II. Comparative Analysis of AODV and R-AODV

\begin{tabular}{|c|c|c|}
\hline PROTOCOL PROPERTY & Avg AODV & $\begin{array}{c}\text { Avg } \\
\text { R-AODV }\end{array}$ \\
\hline Paper/Author Name & Humaira Nishat [18] & Humaira Nishat [18] \\
\hline Throughput & $100 \%$ & $81 \%$ \\
\hline PDR & $88 \%$ & $86 \%$ \\
\hline End to End Delay & $0.18 \mathrm{sec}$ & $0.14 \mathrm{sec}$ \\
\hline NRL & N/A & N/A \\
\hline
\end{tabular}


Sadaf Wali (et-al) A Comprehensive Study on Reactive and Proactive Routing Protocols under different performance Metric

This table is based on the paper of Humaira Nishat [18]. The comparison has been done for two protocols AODV and R-AODV. The mobile nodes are 25 with the packet size of
1500B. The performance of both the protocols is same but with the changes in the network scenarios R-AODV performs low.

TABLE III. Comparative analysis of Routing Protocols

\begin{tabular}{|c|c|c|c|}
\hline $\begin{array}{c}\text { PROTOCOL } \\
\text { PROPERTY }\end{array}$ & Avg AODV & Avg DSR & Avg DSDV \\
\hline Paper/Author Name & A Wajid khan [3] & A Wajid khan [3] & A Wajid khan [3] \\
\hline Throughput & $55 \%$ & $45 \%$ & $45 \%$ \\
\hline PDR & $85 \%$ & $91 \%$ & $86 \%$ \\
\hline End to End Delay & $1.5 \mathrm{sec}$ & $3.0 \mathrm{sec}$ & $0.2 \mathrm{sec}$ \\
\hline NRL & $2.65 \mathrm{sec}$ & $3.0 \mathrm{sec}$ & $0.2 \mathrm{sec}$ \\
\hline
\end{tabular}

The table indicates the result of reactive and proactive routing protocols for different parameters. The results were taken from two papers such as Anuj k [19] and Samir Robert [24]. The simulation parameters used in Anuj $\mathrm{k}$ [19] are as following. The simulation time was $200 \mathrm{sec}$ with the area of 500.500. CBR (UDP) traffic type was used. 500 nodes are used.

Samir Robert [24] the number of nodes used is 10 . This result is for low mobility network. The packet size used is 512 .

\section{Result and Conclusion}

In this survey paper, a comprehensive analysis of reactive and proactive routing protocols under different performance metrics has been done. The different work of the author is also discussed on the bases of the simulation. All routing protocols show good result under specific performance metrics. The average results are shown with the help of graphs and tables of each routing protocols which explains the performance of the reactive and proactive routing protocols in detail.
After comparing all the reactive and proactive routing protocols under different parameters, the overall performance of AODV routing protocol is better than the other protocols.

In the case of throughput, the AODV receives more packets than the other protocols. The NRL of AODV is also lowerssss than the other protocols in different network scenarios. End to end delay and packet delivery ratio (PDR) is also medium in AODV than the other protocols.

\section{Future Work}

To analyze and compare the combination of protocols such as R-AODV and TORA with the other reactive and proactive routing protocols with the different parameters under the network sizes such as small, medium and large. The following parameters such as Throughput, NRL, Jitter, Latency and collision should keep under consideration while comparing the routing protocols. 
Sadaf Wali (et-al) A Comprehensive Study on Reactive and Proactive Routing Protocols under different performance

\section{REFERENCES}

[1].S.Mohapatra, P.K Kanungo "Performance analysis of AODV, DSR, OLSR, and DSDV routing protocols using NS2 Simulator", Proceeded engineering 30(2012) PP 69-76.

[2]. Ajay Prakash Rai, Rasvihari Sharma, Vineet Srivastava, Rashmi Tikar, Rinkoo Bhatia."Performance Comparison of AODV \& DSDV Ad-Hoc Network Routing Protocols On The Basis Of Variable Speed \& Pause Time", International Journal of Engineering and Innovative Technology (IJEIT) Volume 2, Issue 10, April 2013

[3]. Wasi Ullah, Haider Ali, A Wajid Khan, Arshad Farhad, Baseer Ahmad and Adnan Khan, "Performance Assessment of Reactive Routing Protocols in Mobile Ad-hoc Networks under CBR Traffic using NS2", IEEE WiSPNET 2016 conference, PP 1070-1073.

[4]. Akshai Aggarwal, Savita Gandhi, Nirbhay Chaubey." Performance Analysis of AODV, DSDV, and DSR in MANET" international journal of distributed and parallel system (IJDPS) vol.2, No.6, November 2011

[5].Sapna S. Kaushik \& P.R.Deshmukh, "COMPARISON OF EFFECTIVENESS OF AODV, DSDV AND DSR ROUTING PROTOCOLS IN MOBILE AD- HOC NETWORKS", International Journal of Information Technology and Knowledge Management July-December 2009, Volume 2, No. 2, pp. 499-502.

[6].M.Ilyas. "Routing algorithms for balanced energy consumption in Ad-hoc Networks" in the handbook of Ad-Hoc Wireless Networks, 1st ed., 2003.

[7]. C.E. Perkins, E.M. Belding-Royer, and S.Das. "Adhoc on demand distance vector (AODV) routing protocol" RFC 3561, July 2003.
[8].I.D Chakeres.and E.M.Belding Royer "AODV Routing Protocol Implementation Design", IEEE international conference on distributed computing systems workshop (ICDCSW'04),2004.

[9]. V.Rajeshkumar P.Sivakumar, "Comparative study of AODV, DSDV, and DSR routing protocols in Manet using network simulator-2", IEEE international journal of advanced research in computer and communication engineering Volume 2, Issue 12, December 2013, pp 4564-4569

[10]. Zahian Ismail, Rosilah Hassan "Performance of AODV Routing Protocol in Mobile Ad Hoc Network", IEEE 978-1-4244-6716-7/10/\$26.00, 2010.

[11]. Sampo Naski."Performance of Ad Hoc Routing Protocols Characteristics and Comparison", Helsinki University of Technology Telecommunications Software and Multimedia Laboratory HUT T-110.551 Seminar on Internetworking Sjökulla, 2004.

[12]. D. B. Johnson and D. A. Maltz. "Dynamic source routing in Ad-hoc wireless networks". In Imielinski and Korth, editors, Mobile Computing, pages 153181. Kluwer Academic Publishers, 1996.

[13]. David B. Johnson David A. Maltz Josh Broch, "DSR: The Dynamic source routing protocol for multi-hop wireless AdHoc Networks", Computer Science Department Carnegie Mellon University Pittsburgh, PA 15213-3891.

[14]. Mina Vajed Khiavi, Shahram Jamali, Sajjad Jahanbakhsh Gudakahriz "Performance Comparison of AODV, DSDV, DSR and TORA Routing Protocols in MANETs", International 
Sadaf Wali (et-al) A Comprehensive Study on Reactive and Proactive Routing Protocols under different performance

Research Journal of Applied and Basic Sciences. Vol., 3 (7), 1429-1436, 2012.

[15]. Afrah Daas, Khulood Mofleh, Elham Jabr, Sofian Hamad, "Comparison between AODV and DSDV Routing protocols in Mobile Ad-hoc Network (MANET", IEEE 978-1-4799-7626$3 / 15 / \$ 31.00,2015$

[16]. C.E. Perkins and P. Bhagwat, "Highly Dynamic Destination-Sequenced Distance-Vector (DSDV) Routing for Mobile Computers", ACM SIGCOMM Symposium on Communications, Architectures, and Protocols, Sep. 1994, pp. 234-244.

[17]. Mou Zonghuna, Meng Xiaojing. “A Modified AODV Routing protocol based on route stability in MANET",

[18]. Humaira Nishat, Vamsi Krishna K, "Performance Evaluation of On-Demand Routing Protocol AODV and Modified AODV (R-AODV) in MANET" International Journal of Distributed and Parallel Systems (IJDPS) Vol.2, No.1, January 2011.

[19]. Anuj K. Gupta, Dr. Harsh Sadawarti, "Performance analysis of AODV, DSR \& TORA Routing Protocols" IACSIT International Journal of Engineering and Technology, Vol.2, No.2, April 2010 ISSN: 1793-8236.

[20]. Mina Vajed Khiavi, S Shahram Jamali, Sajjad Jahanbakhsh Gudakahriz "Performance Comparison of AODV, DSDV, DSR and TORA Routing Protocols in MANETs" International Research Journal of Applied and Basic Sciences. Vol., 3 (7), 1429-1436, 2012

[21]. Amirhossein Moravejosharieh, Hero Modares, Rosli Salleh, Ehsan Mostajeran "Performance analysis of AODV, AOMDV, DSR, DSDV routing protocols in vehicular ad-hoc network " research journal of recent sciences vol.2(7), 66-73 July (2013)

[22]. Guntupalli Lakshmikanth, Mr. A. Gaiwak, "Simulation-Based Comparative Performance analysis of Adhoc Routing Protocols".

[23]. Geetha Jayakumar, Gopinath Ganapathy "Performance Comparison of MobileAd-hoc Network Routing Protocol" IJCSNS International Journal of Computer Science and Network Security, VOL.7 No.11, November 2007.

[24]. Samir Robert, Rimli Sengupta "comparative performance evaluation of routing protocols for mobile, Ad-hoc networks". 